UDC 519.21

\title{
WAVELET-BASED SIMULATION OF RANDOM PROCESSES FROM CERTAIN CLASSES WITH GIVEN ACCURACY AND RELIABILITY
}

\author{
IEVGEN TURCHYN
}

\begin{abstract}
We consider stochastic processes $Y(t)$ which can be represented as $Y(t)=(X(t))^{s}, s \in \mathbb{N}$, where $X(t)$ is a stationary strictly sub-Gaussian process and build a wavelet-based model that simulates $Y(t)$ with given accuracy and reliability in $L_{p}([0, T])$. A model for simulation with given accuracy and reliability in $L_{p}([0, T])$ is also built for processes $Z(t)$ which can be represented as $Z(t)=X_{1}(t) X_{2}(t)$, where $X_{1}(t)$ and $X_{2}(t)$ are independent stationary strictly sub-Gaussian processes.
\end{abstract}

Key words and phrases. Wavelets, Sub-Gaussian random processes, Simulation.

2010 Mathematics Subject Classification. Primary 60G10; Secondary 42C40.

\section{INTRODUCTION}

Wavelet expansions and wavelet-based expansions form an interesting class of representations of random processes. At present there exist many articles devoted to such expansions and their properties, some of them are [1], [3], [4], [8], [9] and [14]-[16]. Wavelet-based expansions with uncorrelated terms (see, for instance, articles [4], [16]) are especially important since they are very convenient for approximation and simulation of random processes.

We will consider simulation of stochastic processes with given accuracy and reliability. This is simulation of a random process by a model which has guaranteed rate of convergence in a certain sense - i.e., a model $\hat{X}(t)$ approximates a process $X(t)$ with given accuracy $\varepsilon$ and reliability $1-\delta$ in a functional space $U(\mathbf{T})$ if

$$
P\left\{\|X-\hat{X}\|_{U(\mathbf{T})}>\varepsilon\right\} \leq \delta .
$$

Simulation of stochastic processes with given accuracy and reliability has been studied, in particular, in [11] (see also e.g. [5], [10], [13] and [14]). It is necessary to mention that results on simulation with given accuracy and reliability are available mostly for light-tailed processes - Gaussian and sub-Gaussian processes (although there are some exceptions, see, for instance, [18]).

The article is devoted to simulation with given accuracy and reliability in $L_{p}([0, T])$ of random processes which can be represented as

$$
Y(t)=(X(t))^{s}, s \in \mathbb{N},
$$

where $X(t)$ is a stationary strictly sub-Gaussian process, and

$$
Z(t)=X_{1}(t) X_{2}(t)
$$

where $X_{1}(t)$ and $X_{2}(t)$ are stationary strictly sub-Gaussian processes. So our approach allows simulation of processes with one-dimensional distributions which have relatively heavy tails (i.e. heavier than the Gaussian ones).

Our models are derived from a model which was studied in [14], where a waveletbased expansion was considered and used for construction of a model of a process (this or similar wavelet-based expansions and their rate of convergence were also studied in [4], [20] and [21]). 


\section{Sub-Gaussian RANDOM VARIABLES AND PROCESSES}

Definition 2.1. [2] Let $\{\Omega, \mathfrak{F}, \mathrm{P}\}$ be a standard probability space. A random variable $\xi$ is called sub-Gaussian if

1) $\mathrm{E} \xi=0$;

2) for all $\lambda \in \mathbb{R}$ there exists $E \exp \{\lambda \xi\}$;

3) there exists such constant $a>0$ that inequality

$$
\mathrm{E} \exp \{\lambda \xi\} \leq \exp \left\{a^{2} \lambda^{2} / 2\right\}
$$

holds for all $\lambda \in \mathbb{R}$.

We will denote the set of all sub-Gaussian random variables by $\operatorname{Sub}(\Omega)$. $\operatorname{Sub}(\Omega)$ is a Banach space with respect to the norm

$$
\tau(\xi)=\sup _{\lambda \neq 0}\left(\frac{2 \ln E \exp \{\lambda \xi\}}{\lambda^{2}}\right)^{1 / 2}
$$

(see [2]). Examples of sub-Gaussian random variables can be found in [2]. Let us note that centered normal random variables belong to $\operatorname{Sub}(\Omega)$.

Definition 2.2. [2] A sub-Gaussian random variable $\xi$ is called strictly sub-Gaussian if $\tau(\xi)=\left(E \xi^{2}\right)^{1 / 2}$.

Definition 2.3. [2] A family of sub-Gaussian random variables $\Delta$ is called strictly subGaussian if for any finite or countable set $\left\{\xi_{i}, i \in I\right\}$ from $\Delta$ and all $\lambda_{i} \in \mathbb{R}$ holds relation

$$
\tau^{2}\left(\sum_{i \in I} \lambda_{i} \xi_{i}\right)=\mathrm{E}\left(\sum_{i \in I} \lambda_{i} \xi_{i}\right)^{2}
$$

Definition 2.4. [2] A stochastic process $X=\{X(t), t \in \mathbf{T}\}$ is called strictly subGaussian if the family of random variables $\{X(t), t \in \mathbf{T}\}$ is strictly sub-Gaussian.

Example 2.1. [2] Let $X=\{X(t), t \in \mathbf{T}\}$ be a centered Gaussian process. Then $X(t)$ is a strictly sub-Gaussian stochastic process.

Example 2.2. Let $X=\{X(t), t \in \mathbf{T}\}$ be a random process such that

$$
X(t)=\sum_{k=1}^{\infty} \xi_{k} f_{k}(t),
$$

where $\xi=\left\{\xi_{k}, k=1,2, \ldots\right\}$ is a family of independent strictly sub-Gaussian random variables and for all $t \in \mathbf{T}$

$$
\sum_{k=1}^{\infty} \mathrm{E} \xi_{k}^{2} f_{k}^{2}(t)<\infty
$$

Then $X(t)$ is a strictly sub-Gaussian stochastic process.

\section{EXPANSION OF A RANDOM PROCESS INTO A WAVELET-BASED SERIES}

Definition 3.1. [6] Let $\phi \in L_{2}(\mathbb{R})$ be such a function that the following assumptions hold:

i)

$$
\sum_{k \in \mathbb{Z}}|\hat{\phi}(y+2 \pi k)|^{2}=1
$$

almost everywhere, where $\hat{\phi}(y)$ is the Fourier transform of $\phi$; 
ii) There exists a function $m_{0} \in L_{2}([0,2 \pi])$ such that $m_{0}(x)$ has period $2 \pi$ and almost everywhere

$$
\hat{\phi}(y)=m_{0}\left(\frac{y}{2}\right) \hat{\phi}\left(\frac{y}{2}\right) ;
$$

iii) $\hat{\phi}(0) \neq 0$ and the function $\hat{\phi}(y)$ is continuous at 0 .

The function $\phi(x)$ is called $f$-wavelet. Let $\psi(x)$ be the inverse Fourier transform of the function

$$
\hat{\psi}(y)=\overline{m_{0}\left(\frac{y}{2}+\pi\right)} \exp \left\{-i \frac{y}{2}\right\} \hat{\phi}\left(\frac{y}{2}\right) .
$$

The function $\psi(x)$ is called $m$-wavelet.

Let

$$
\phi_{j k}(x)=2^{j / 2} \phi\left(2^{j} x-k\right), \quad \psi_{j k}(x)=2^{j / 2} \psi\left(2^{j} x-k\right), \quad k \in \mathbb{Z}, \quad j=0,1,2, \ldots
$$

The family of functions $\left\{\phi_{0 k}, \psi_{j k}, j=0,1, \ldots, k \in \mathbb{Z}\right\}$ is an orthonormal basis in $L_{2}(\mathbb{R})$ (see, for example, [6]).

Remark 3.1. We will consider only real-valued wavelets below.

Let us now formulate a result which is very important for us.

Theorem 3.1. [12] Suppose that $X(t), t \in \mathbb{R}$, is a centered second-order random process such that its correlation function $R(t, s)=\mathrm{E} X(t) \overline{X(s)}$ can be represented as

$$
R(t, s)=\int_{\mathbb{R}} u(t, y) \overline{u(s, y)} d y,
$$

where $u(t, y)$ is a Borel function which belongs to $L_{2}(\mathbb{R})$ for all $t \in \mathbb{R},\left\{\phi_{0 k}(x)\right.$, $\left.\psi_{j k}(x), \quad k \in \mathbb{Z}, j=0,1, \ldots\right\}$ is an arbitrary wavelet basis,

$$
\begin{aligned}
& a_{0 k}(t)=\frac{1}{\sqrt{2 \pi}} \int_{\mathbb{R}} u(t, y) \overline{\hat{\phi}_{0 k}(y)} d y, \\
& b_{j k}(t)=\frac{1}{\sqrt{2 \pi}} \int_{\mathbb{R}} u(t, y) \overline{\hat{\psi}_{j k}(y)} d y,
\end{aligned}
$$

$\hat{\phi}_{0 k}(y)$ and $\hat{\psi}_{j k}(y)$ are the Fourier transforms of $\phi_{0 k}(y)$ and $\psi_{j k}(y)$ respectively.

Then

$$
X(t)=\sum_{k \in \mathbb{Z}} \xi_{0 k} a_{0 k}(t)+\sum_{j=0}^{\infty} \sum_{k \in \mathbb{Z}} \eta_{j k} b_{j k}(t),
$$

series (4) converges in $L_{2}(\Omega)$ for all $t \in \mathbb{R}$, where $\xi_{0 k}, \eta_{j k}$ are centered random variables such that

$$
\mathrm{E} \xi_{0 k} \overline{\xi_{0 l}}=\delta_{k l}, \quad \mathrm{E} \eta_{j k} \overline{\eta_{l m}}=\delta_{j l} \delta_{k m}, \quad \mathrm{E} \xi_{0 k} \overline{\eta_{n l}}=0 .
$$

Corollary 3.1. [12] Suppose that a centered second-order stationary process $X(t)$ has the spectral density $f(y),\left\{\phi_{0 k}(x), \psi_{j k}(x), k \in \mathbb{Z}, j=0,1, \ldots\right\}$ is a wavelet basis, $g(y)=\sqrt{f(y)}$. Then $X(t)$ can be represented as a mean square convergent series (4) and

$$
\begin{gathered}
a_{0 k}(t)=\frac{1}{\sqrt{2 \pi}} \int_{\mathbb{R}} g(y) \exp \{-i y(t-k)\} \overline{\hat{\phi}(y)} d y, \\
b_{j k}(t)=\frac{1}{\sqrt{2 \pi} 2^{j / 2}} \int_{\mathbb{R}} g(y) \exp \left\{-i y\left(t-\frac{k}{2^{j}}\right)\right\} \overline{\hat{\psi}\left(y / 2^{j}\right)} d y,
\end{gathered}
$$

where the random variables $\xi_{0 k}, \eta_{j k}$ from (4) are such that

$$
\begin{gathered}
\mathrm{E} \xi_{0 k}=\mathrm{E} \eta_{j k}=0, \\
\mathrm{E} \xi_{0 k} \overline{\xi_{0 l}}=\delta_{k l}, \quad \mathrm{E} \eta_{j k} \overline{\eta_{l m}}=\delta_{j l} \delta_{k m}, \quad \mathrm{E} \xi_{0 k} \overline{\eta_{n l}}=0 .
\end{gathered}
$$




\section{Simulation with given accuracy and Reliability in $L_{p}([0, T])$}

By a stationary process we will always mean a wide-sense stationary process below.

Definition 4.1. Suppose that a stationary random process $X=\{X(t), t \in \mathbb{R}\}$ satisfies the conditions of Corollary 3.1. We call the following process a model of $X(t)$ :

$$
\hat{X}(t)=\sum_{k=-\left(N_{0}-1\right)}^{N_{0}-1} \xi_{0 k} a_{0 k}(t)+\sum_{j=0}^{N-1} \sum_{k=-\left(M_{j}-1\right)}^{M_{j}-1} \eta_{j k} b_{j k}(t),
$$

where $\xi_{0 k}, \eta_{j k}$ are the random variables from the expansion (4), $a_{0 k}(t)$ and $b_{j k}(t)$ are calculated using formulae (5) and (6), $N_{0}>1, N>1, M_{j}>1(j=0,1, \ldots, N-1)$.

Numerical characteristics which describe the rate of approximation of a process by its model are accuracy and reliability.

Definition 4.2. We say that a model $\hat{X}(t)$ approximates a process $X(t)$ with given reliability $1-\delta(0<\delta<1)$ and accuracy $\varepsilon>0$ in $L_{p}([0, T]), p>0$, if

$$
P\left\{\left(\int_{0}^{T}|X(t)-\hat{X}(t)|^{p}\right)^{1 / p}>\varepsilon\right\} \leq \delta .
$$

4.1. Simulation of $\mathbf{Y}(\mathbf{t})=(\mathbf{X}(\mathbf{t}))^{\mathbf{s}}$. If $\hat{X}(t)$ is a model for a process $X(t)$ then a natural model for a process $Y(t)=F(X(t))$ is a "plug-in" model $\hat{Y}(t)=F(\hat{X}(t))$. So we will use $\hat{Y}(t)=(\hat{X}(t))^{s}$ as a model for $Y(t)=(X(t))^{s}$.

Theorem 4.1. Let $Y(t)=(X(t))^{s}, t \in \mathbb{R}, s \in \mathbb{N}$, where $X(t), t \in \mathbb{R}$, is a mean square continuous stationary strictly sub-Gaussian stochastic process which has spectral density $f(y), g(y)=\sqrt{f(y)}, R(\tau)$ is the correlation function of $X(t), \phi$ is a $f$-wavelet, $\psi$ is the corresponding $m$-wavelet. Let the random variables $\xi_{0 k}, \eta_{j k}$ in the expansion (4) of $X(t)$ be independent and strictly sub-Gaussian. Suppose that the following conditions hold: there exist the derivatives $g^{\prime}(y), \hat{\psi}^{\prime}(y), \hat{\phi}^{\prime}(y),|\hat{\psi}(y)|<C_{1},\left|\hat{\psi}^{\prime}(y)\right|<C_{2}, f(y) \rightarrow 0$ as $|y| \rightarrow \infty, g(y)$ and $\hat{\phi}(y)$ are absolutely continuous,

$$
\begin{gathered}
\sup _{y \in \mathbb{R}}|\hat{\phi}(y)|<\infty, \quad \sup _{y \in \mathbb{R}} g(y)<\infty, \\
\int_{\mathbb{R}} g(y) d y<\infty, \quad \int_{\mathbb{R}}\left|g^{\prime}(y)\right||y| d y<\infty, \\
\int_{\mathbb{R}} g(y)|y| d y<\infty \quad \int_{\mathbb{R}}\left|g^{\prime}(y)\right||\hat{\phi}(y)| d y<\infty, \\
\int_{\mathbb{R}} g(y)\left|\hat{\phi}^{\prime}(y)\right| d y<\infty .
\end{gathered}
$$

Denote

$$
\begin{gathered}
A=\frac{C_{2}}{\sqrt{2 \pi}} \int_{\mathbb{R}}\left(\left|g^{\prime}(y)\right||y|+g(y)\right) d y, \\
B=\frac{C_{2}}{\sqrt{2 \pi}} \int_{\mathbb{R}} g(y)|y| d y, \\
A_{1}=\frac{1}{\sqrt{2 \pi}} \int_{\mathbb{R}}\left(\left|g^{\prime}(y)\right||\hat{\phi}(y)|+g(y)\left|\hat{\phi}^{\prime}(y)\right|\right) d y, \\
B_{1}=\frac{1}{\sqrt{2 \pi}} \int_{\mathbb{R}} g(y)|\hat{\phi}(y)| d y .
\end{gathered}
$$

Let $p \geq 2, T>0, \varepsilon>0,0<\delta<1$ and let the model $\hat{X}(t)$ of $X(t)$ be defined by (7). Set $\hat{Y}(t)=(\hat{X}(t))^{s}$. 
If

$$
\begin{gathered}
N_{0}>\frac{6}{\delta_{1}}\left(A_{1}+B_{1} T\right)^{2}+1, \\
N>\max \left\{1+\log _{2}\left(\frac{72(A+B T)^{2}}{5 \delta_{1}}\right), 1+\log _{8}\left(\frac{18 B^{2}}{7 \delta_{1}}\right)\right\}, \\
M_{j}>1+\frac{12}{\delta_{1}}(A+B T)^{2}\left(1-\frac{1}{2^{N}}\right), \quad j=0,1, \ldots, N-1,
\end{gathered}
$$

where

$$
\begin{gathered}
\delta_{1}=\frac{\delta^{2 / p} \mathcal{\varepsilon}^{2}}{\left(D^{*}\right)^{2 / p}}, \\
D^{*}=2^{(p s+3) / 2} T p \sqrt{\Gamma(p)} s^{p-1 / 2}(R(0))^{p(s-1) / 2} \\
\cdot\left[(s-1) \Gamma(p(s-1))+\sum_{k=1}^{s-2} \sqrt{k(s-1-k) \Gamma(2 p k) \Gamma(2 p(s-1-k))}\right]^{1 / 2},
\end{gathered}
$$

then the model $\hat{Y}(t)$ approximates the process $Y(t)$ with given accuracy $\varepsilon$ and reliability $1-\delta$ in $L_{p}([0, T])$.

Proof. Denote $\Delta X(t)=X(t)-\hat{X}(t), \Delta Y(t)=Y(t)-\hat{Y}(t)$,

$$
\|\Delta Y\|_{p}=\left(\int_{0}^{T}|\Delta Y(t)|^{p} d t\right)^{1 / p}
$$

According to Lemma 4.1 from [14] the following inequalities hold under the conditions of the theorem:

$$
\begin{gathered}
\left|a_{0 k}(t)\right| \leq \frac{A_{1}+B_{1}|t|}{|k|}, k \neq 0, \\
\left|b_{j 0}(t)\right| \leq \frac{B}{2^{3 j / 2}}, \\
\left|b_{j k}(t)\right| \leq \frac{A+B|t|}{|k| 2^{j / 2}}, k \neq 0 .
\end{gathered}
$$

It follows from (8)-(10) and (11)-(13) that

$$
\sup _{t \in[0, T]} \mathrm{E}|\Delta X(t)|^{2} \leq \delta_{1} .
$$

We will need the following inequality (see [17]): if $\xi$ is a sub-Gaussian random variable, then

$$
\mathrm{E}|\xi|^{p} \leq p 2^{p / 2}(\tau(\xi))^{p} \Gamma(p / 2), p>0
$$

Let us estimate $\mathrm{E}\|\Delta Y\|_{p}^{2}$. Using the Lyapunov inequality, we have:

$$
\mathrm{E}\|\Delta Y\|_{p}^{2}=\mathrm{E}\left(\int_{0}^{T}|Y(t)-\hat{Y}(t)|^{p} d t\right)^{2 / p} \leq\left(\int_{0}^{T} \mathrm{E}|Y(t)-\hat{Y}(t)|^{p} d t\right)^{2 / p} .
$$

Applying the Cauchy-Schwarz inequality we obtain:

$$
\begin{gathered}
\mathrm{E}|Y(t)-\hat{Y}(t)|^{p}=\mathrm{E}\left|(X(t))^{s}-(\hat{X}(t))^{s}\right|^{p} \\
=\mathrm{E}|X(t)-\hat{X}(t)|^{p}\left|\sum_{k=0}^{s-1}(X(t))^{k}(\hat{X}(t))^{s-1-k}\right|^{p} \\
\leq\left(\mathrm{E}|X(t)-\hat{X}(t)|^{2 p}\right)^{1 / 2}\left(\mathrm{E}\left|\sum_{k=0}^{s-1}(X(t))^{k}(\hat{X}(t))^{s-1-k}\right|^{2 p}\right)^{1 / 2} .
\end{gathered}
$$


It follows from (14) that

$$
\left(\mathrm{E}|X(t)-\hat{X}(t)|^{2 p}\right)^{1 / 2} \leq\left(p 2^{p+1} \Gamma(p)\right)^{1 / 2}(\sigma(\Delta X(t)))^{p} .
$$

Since

$$
\mathrm{E}\left|\zeta_{1}+\zeta_{2}+\ldots+\zeta_{s}\right|^{2 p} \leq s^{2 p-1}\left(\mathrm{E}\left|\zeta_{1}\right|^{2 p}+\mathrm{E}\left|\zeta_{2}\right|^{2 p}+\ldots+\mathrm{E}\left|\zeta_{s}\right|^{2 p}\right)
$$

(an application of the power mean inequality) we have

$$
\mathrm{E}\left|\sum_{k=0}^{s-1}(X(t))^{k}(\hat{X}(t))^{s-1-k}\right|^{2 p} \leq s^{2 p-1} \sum_{k=0}^{s-1} \mathrm{E}|X(t)|^{2 p k}|\hat{X}(t)|^{2 p(s-1-k)} .
$$

But using (14) and Cauchy-Schwarz inequality we obtain

$$
\mathrm{E}|X(t)|^{2 p k}|\hat{X}(t)|^{2 p(s-1-k)} \leq C_{k}^{*}(R(0))^{p(s-1)}, \quad 1 \leq k \leq s-2,
$$

where

$$
C_{k}^{*}=4 p 2^{p(s-1)} \sqrt{k(s-1-k) \Gamma(2 p k) \Gamma(2 p(s-1-k))}
$$

(we used the inequality $\sigma(\hat{X}(t))<\sigma(X(t))$ ).

It follows from (14) that

$$
\begin{aligned}
& \mathrm{E}|X(t)|^{2 p(s-1)} \leq 2 p(s-1) 2^{p(s-1)} \Gamma(p(s-1))(R(0))^{p(s-1)}, \\
& \mathrm{E}|\hat{X}(t)|^{2 p(s-1)} \leq 2 p(s-1) 2^{p(s-1)} \Gamma(p(s-1))(R(0))^{p(s-1)} .
\end{aligned}
$$

Using (17)-(20) we have

$$
\int_{0}^{T} \mathrm{E}|Y(t)-\hat{Y}(t)|^{p} d t \leq \delta_{1}^{p / 2} D^{*}=\delta \varepsilon^{p}
$$

Applying the Markov inequality we get

$$
P\left\{\|\Delta Y\|_{p}>\varepsilon\right\}=P\left\{\|\Delta Y\|_{p}^{p}>\varepsilon^{p}\right\} \leq \delta .
$$

So the theorem is proved.

Example 4.1. A stationary centered Gaussian process $X=\{X(t), t \in \mathbb{R}\}$ with spectral density $f(y)=\frac{1}{\left(1+y^{2 n}\right)^{2}}, n \geq 2$, and an arbitrary Daubechies wavelet satisfy the conditions of Theorem 4.1 .

4.2. Simulation of $\mathbf{Z}(\mathbf{t})=\mathbf{X}_{\mathbf{1}}(\mathbf{t}) \mathbf{X}_{\mathbf{2}}(\mathbf{t})$. Let us now consider a stochastic process $Z(t)$ which can be represented as

$$
Z(t)=X_{1}(t) X_{2}(t)
$$

where $X_{1}(t)$ and $X_{2}(t)$ are independent stationary strictly sub-Gaussian stochastic processes which have spectral densities $f_{1}(y)$ and $f_{2}(y)$ correspondingly. Let $\phi_{1}, \psi_{1}$ and $\phi_{2}, \psi_{2}$ be two pairs of a $f$-wavelet and the corresponding $m$-wavelet.

According to Corollary 3.1, the processes $X_{1}(t)$ and $X_{2}(t)$ can be expanded as

$$
\begin{aligned}
& X_{1}(t)=\sum_{k \in \mathbb{Z}} \xi_{0 k}^{(1)} a_{0 k}^{(1)}(t)+\sum_{j=0}^{\infty} \sum_{k \in \mathbb{Z}} \eta_{j k}^{(1)} b_{j k}^{(1)}(t), \\
& X_{2}(t)=\sum_{k \in \mathbb{Z}} \xi_{0 k}^{(2)} a_{0 k}^{(2)}(t)+\sum_{j=0}^{\infty} \sum_{k \in \mathbb{Z}} \eta_{j k}^{(2)} b_{j k}^{(2)}(t),
\end{aligned}
$$

where

$$
\begin{gathered}
a_{0 k}^{(s)}(t)=\frac{1}{\sqrt{2 \pi}} \int_{\mathbb{R}} g_{s}(y) \exp \{-i y(t-k)\} \overline{\hat{\phi}_{s}(y)} d y, \\
b_{j k}^{(s)}(t)=\frac{1}{\sqrt{2 \pi} 2^{j / 2}} \int_{\mathbb{R}} g_{s}(y) \exp \left\{-i y\left(t-\frac{k}{2^{j}}\right)\right\} \overline{\hat{\psi}_{s}\left(y / 2^{j}\right)} d y,
\end{gathered}
$$


$g_{s}(y)=\sqrt{f_{s}(y)}$, random variables $\xi_{0 k}^{(s)}, \eta_{j l}^{(s)}$ are uncorrelated,

$$
\mathrm{E}\left|\xi_{0 k}^{(s)}\right|^{2}=1, \mathrm{E}\left|\eta_{j l}^{(s)}\right|^{2}=1
$$

$s=1,2$.

We will consider a "plug-in" model

$$
\hat{Z}(t)=\hat{X}_{1}(t) \hat{X}_{2}(t)
$$

for the process $Z(t)$, where $\hat{X}_{1}(t)$ and $\hat{X}_{2}(t)$ are models of type $(7)$ for $X_{1}(t)$ and $X_{2}(t)$ correspondingly, i.e.

$$
\hat{X}_{s}(t)=\sum_{k=-\left(N_{0}^{(s)}-1\right)}^{N_{0}^{(s)}-1} \xi_{0 k}^{(s)} a_{0 k}^{(s)}(t)+\sum_{j=0}^{N^{(s)}-1} \sum_{k=-\left(M_{j}^{(s)}-1\right)}^{M_{j}^{(s)}-1} \eta_{j k}^{(s)} b_{j k}^{(s)}(t),
$$

$N_{0}^{(s)}>1, N^{(s)}>1, M_{j}^{(s)}>1\left(j=0,1, \ldots, N^{(s)}-1\right), s=1,2$.

Theorem 4.2. Let $Z(t)=X_{1}(t) X_{2}(t), t \in \mathbb{R}$, where $X_{1}(t), X_{2}(t), t \in \mathbb{R}$, are mean square continuous stationary strictly sub-Gaussian stochastic processes which have spectral densities $f_{s}(y), g_{s}(y)=\sqrt{f_{s}(y)}, R_{s}(\tau)$ is the correlation function of $X_{s}(t)(s=1,2), \phi_{1}, \psi_{1}$ and $\phi_{2}, \psi_{2}$ are two pairs of a $f$-wavelet and the corresponding $m$-wavelet. Let the random variables $\xi_{0 k}^{(s)}, \eta_{j k}^{(s)}(s=1,2)$ in expansions $(21),(22)$ of $X_{1}(t)$ and $X_{2}(t)$ be independent and strictly sub-Gaussian. Suppose that the following conditions hold: there exist derivatives $g_{s}^{\prime}(y), \hat{\psi}_{s}^{\prime}(y), \hat{\phi}_{s}^{\prime}(y),\left|\hat{\psi}_{s}(y)\right|<C_{1}^{(s)},\left|\hat{\psi}_{s}^{\prime}(y)\right|<C_{2}^{(s)}, f_{s}(y) \rightarrow 0$ as $|y| \rightarrow \infty, g_{s}(y)$ and $\hat{\phi}_{s}(y)$ are absolutely continuous,

$$
\begin{gathered}
\sup _{y \in \mathbb{R}}\left|\hat{\phi}_{s}(y)\right|<\infty, \quad \sup _{y \in \mathbb{R}} g_{s}(y)<\infty, \\
\int_{\mathbb{R}} g_{s}(y) d y<\infty, \quad \int_{\mathbb{R}}\left|g_{s}^{\prime}(y)\right||y| d y<\infty, \\
\int_{\mathbb{R}} g_{s}(y)|y| d y<\infty \quad \int_{\mathbb{R}}\left|g_{s}^{\prime}(y)\right|\left|\hat{\phi}_{s}(y)\right| d y<\infty, \\
\int_{\mathbb{R}} g_{s}(y)\left|\hat{\phi}_{s}^{\prime}(y)\right| d y<\infty,
\end{gathered}
$$

$s=1,2$. Denote

$$
\begin{gathered}
A^{(s)}=\frac{C_{2}^{(s)}}{\sqrt{2 \pi}} \int_{\mathbb{R}}\left(\left|g_{s}^{\prime}(y)\right||y|+g_{s}(y)\right) d y, \\
B^{(s)}=\frac{C_{2}^{(s)}}{\sqrt{2 \pi}} \int_{\mathbb{R}} g_{s}(y)|y| d y, \\
A_{1}^{(s)}=\frac{1}{\sqrt{2 \pi}} \int_{\mathbb{R}}\left(\left|g_{s}^{\prime}(y)\right|\left|\hat{\phi}_{s}(y)\right|+g_{s}(y)\left|\hat{\phi}_{s}^{\prime}(y)\right|\right) d y, \\
B_{1}^{(s)}=\frac{1}{\sqrt{2 \pi}} \int_{\mathbb{R}} g_{s}(y)\left|\hat{\phi}_{s}(y)\right| d y,
\end{gathered}
$$

$s=1,2$.

Let $p \geq 1, T>0, \varepsilon>0,0<\delta<1$ and let the models $\hat{Z}(t), \hat{X}_{s}(t)$ of $Z(t), X_{s}(t)$ $(s=1,2)$ be defined by $(25),(26)$ correspondingly.

If

$$
\begin{gathered}
N_{0}^{(s)}>\frac{6}{\delta_{s}^{*}}\left(A_{1}^{(s)}+B_{1}^{(s)} T\right)^{2}+1, \\
N^{(s)}>\max \left\{1+\log _{2}\left(\frac{72\left(A^{(s)}+B^{(s)} T\right)^{2}}{5 \delta_{s}^{*}}\right), 1+\log _{8}\left(\frac{18\left(B^{(s)}\right)^{2}}{7 \delta_{s}^{*}}\right)\right\},
\end{gathered}
$$




$$
M_{j}^{(s)}>1+\frac{12}{\delta_{s}^{*}}\left(A^{(s)}+B^{(s)} T\right)^{2}\left(1-\frac{1}{2^{N^{(s)}}}\right), \quad j=0,1, \ldots, N^{(s)}-1,
$$

$s=1,2$, where

$$
\begin{gathered}
\delta_{1}^{*}=\hat{\delta} / R_{2}(0), \delta_{2}^{*}=\hat{\delta} / R_{1}(0), \\
\hat{\delta}=\frac{\delta^{2 / p} \varepsilon^{2}}{\left(2^{2 p+1} p \Gamma(p) T\right)^{2 / p}},
\end{gathered}
$$

then the model $\hat{Z}(t)$ defined by (25) approximates the process $Z(t)$ with given accuracy $\varepsilon$ and reliability $1-\delta$ in $L_{p}([0, T])$.

Proof. Denote $\Delta X_{s}(t)=X_{s}(t)-\hat{X}_{s}(t)$,

$$
\left\|\Delta X_{s}\right\|_{p}=\left(\int_{0}^{T}\left|\Delta X_{s}(t)\right|^{p} d t\right)^{1 / p}
$$

$s=1,2$. Let us estimate

$$
\mathrm{E}\left\|X_{1} X_{2}-\hat{X}_{1} \hat{X}_{2}\right\|_{p}
$$

Applying (14), the Cauchy-Schwarz inequality and the power mean inequality we get

$$
\begin{gathered}
\mathrm{E}\left|X_{1}(t) X_{2}(t)-\hat{X}_{1}(t) \hat{X}_{2}(t)\right|^{p} \\
\leq 2^{p-1}\left(\mathrm{E}\left|X_{2}(t)\left(X_{1}(t)-\hat{X}_{1}(t)\right)\right|^{p}+\mathrm{E}\left|\hat{X}_{1}(t)\left(X_{2}(t)-\hat{X}_{2}(t)\right)\right|^{p}\right) \\
\leq 2^{p-1}\left(\left(\mathrm{E}\left|X_{2}(t)\right|^{2 p}\right)^{1 / 2}\left(\mathrm{E}\left|X_{1}(t)-\hat{X}_{1}(t)\right|^{2 p}\right)^{1 / 2}\right. \\
\left.+\left(\mathrm{E}\left|\hat{X}_{1}(t)\right|^{2 p}\right)^{1 / 2}\left(\mathrm{E}\left|X_{2}(t)-\hat{X}_{2}(t)\right|^{2 p}\right)^{1 / 2}\right) \\
\leq 2^{p-1}\left(C_{R}(p) \sigma^{p}\left(X_{2}(t)\right) \sigma^{p}\left(X_{1}(t)-\hat{X}_{1}(t)\right)+C_{R}(p) \sigma^{p}\left(X_{1}(t)\right) \sigma^{p}\left(X_{2}(t)-\hat{X}_{2}(t)\right)\right),
\end{gathered}
$$

where

It follows from (27)-(29) that

$$
C_{R}(p)=2 p 2^{p} \Gamma(p)
$$

$$
\sup _{t \in[0 ; T]} \sigma^{2}\left(\Delta X_{s}(t)\right) \leq \delta_{s}^{*}
$$

$s=1,2$. Now we see that

$$
\begin{gathered}
\int_{0}^{T} \mathrm{E}|Z(t)-\hat{Z}(t)|^{p} d t \\
\leq 2^{p-1} C_{R}(p) T\left(\left(R_{2}(0)\right)^{p / 2}\left(\sup _{t \in[0, T]} \sigma^{2}\left(\Delta X_{1}(t)\right)\right)^{p / 2}+\left(R_{1}(0)\right)^{p / 2}\left(\sup _{t \in[0, T]} \sigma^{2}\left(\Delta X_{2}(t)\right)\right)^{p / 2}\right) \\
\leq \delta \varepsilon^{p}
\end{gathered}
$$

and, using the Markov inequality, we obtain

$$
P\left\{\|Z-\hat{Z}\|_{p}>\varepsilon\right\} \leq \delta .
$$

Example 4.2. Let us consider a process $Z(t)=X_{1}(t) X_{2}(t)$, where $X_{1}(t)$ and $X_{2}(t)$ are independent centered stationary Gaussian stochastic processes which have spectral densities

and

$$
f_{1}(y)=\frac{1}{\left(1+y^{2}\right)^{2 n}}, n \geq 2
$$

$$
f_{2}(y)=\left(\frac{1}{\left(1+(y-a)^{2}\right)^{m}}+\frac{1}{\left(1+(y+a)^{2}\right)^{m}}\right)^{2}, m \geq 2,
$$


correspondingly. Let us take as $\phi_{1}, \phi_{2}$ and $\psi_{1}, \psi_{2}$ correspondingly two Daubechies $f$ wavelets and $m$-wavelets of any order. These two pairs of a process and the corresponding wavelet satisfy the conditions of the theorem.

\section{Conclusions}

We built a wavelet-based model for simulation of a process which is an integer power of a sub-Gaussian process. A wavelet-based model was also built for a process $Z(t)$ which can be represented as $Z(t)=X_{1}(t) X_{2}(t)$, where $X_{1}(t)$ and $X_{2}(t)$ are stationary strictly sub-Gaussian processes.

We proved theorems about simulation of stochastic processes by the above-mentioned models with given accuracy and reliability in $L_{p}([0 ; T])$.

The author expresses gratitude to professor Yury Kozachenko for valuable discussions.

\section{REFERENCES}

1. A. Ayache, M. S. Taqqu, Rate optimality of wavelet series approximations of fractional Brownian motion, J. Fourier Anal. Appl. 9 (2003), no. 5, 451-471.

2. V. V. Buldygin, Yu. V. Kozachenko, Metric Characterization of Random Variables and Random Processes, Amer. Math. Soc., Providence, RI, 2000.

3. S. Cambanis, E. Masry, Wavelet approximation of deterministic and random signals: convergence properties and rates, IEEE Trans. Inform. Theory, 40 (1994), no. 4, 1013-1029.

4. G. Didier, V. Pipiras, Gaussian stationary processes: adaptive wavelet decompositions, discrete approximations, and their convergence, J. Fourier Anal. Appl. 14 (2008), no. 2, 203-234.

5. B. V. Dovgay, Yu. V. Kozachenko, I. V. Rozora, Simulation of Random Processes in Physical Systems, Zadruga, Kyiv, 2010. (Ukrainian)

6. W. Härdle, G. Kerkyacharian, D. Picard, A. Tsybakov, Wavelets, Approximation and Statistical Applications, Springer, N.Y., 1998.

7. E. Hernández, G. Weiss, A First Course on Wavelets, CRC Press Inc., Boca Rotan, FL, 1996.

8. Y. Kozachenko, A. Olenko, O. Polosmak, Uniform convergence of wavelet expansions of Gaussian random processes, Stoch. Anal. Appl. 29 (2011), no. 2, 169-184.

9. Y. Kozachenko, A. Olenko, O. Polosmak, Uniform convergence of compactly supported wavelet expansions of Gaussian random processes, Comm. Statist. Theory Methods 43 (2014), no. 10-12, 2549-2562.

10. Yu. Kozachenko, O. Pogoriliak, Simulation of Cox processes driven by random Gaussian field, Methodol. Comput. Appl. Probab. 13 (2011), 511-521.

11. Yu. Kozachenko, O. Pogorilyak, I. Rozora, A. Tegza, Simulation of Stochastic Processes with Given Accuracy and Reliability, ISTE Press, London, 2016.

12. Yu. V. Kozachenko, I. V. Rozora, Ye. V. Turchyn, On an expansion of random processes in series, Random Oper. Stoch. Equ. 15 (2007), no. 1, 15-33.

13. Yu. Kozachenko, T. Sottinen, O. Vasylyk, Simulation of weakly self-similar stationary increment $\operatorname{Sub}_{\varphi}(\Omega)$-processes: a series expansion approach, Methodol. Comput. Appl. Probab. 7 (2005), 379-400.

14. Yu. Kozachenko, Ye. Turchyn, On Karhunen-Loeve-like expansion for a class of random processes, Int. J. Stat. Manag. Syst. 3 (2008), 43-55.

15. E. Masry, Convergence properties of wavelet series expansions of fractional Brownian motion, Appl. Comput. Harmon. Anal. 3 (1996), no. 3, 239-253.

16. Y. Meyer, F. Sellan, M. S. Taqqu, Wavelets, generalized white noise and fractional integration: the synthesis of fractional Brownian motion, J. Fourier Anal. Appl. 5 (1999), no. 5, 465-494.

17. O. Rivasplata, Subgaussian random variables: An expository note, DOI: 10.13140 /RG.2.2.36288.23040

18. I. Turchyn, A multiplicative wavelet-based model for simulation of a random process, Mod. Stoch. Theory Appl. 2 (2015), 309-325.

19. Y. Turchyn, Simulation of sub-Gaussian processes using wavelets, Monte Carlo Methods Appl. 17 (2011), 215-231.

20. G. Walter, J. Zhang, A wavelet-based KL-like expansion for wide-sense stationary random processes, IEEE Trans. Signal Process. 42 (1994), no. 7, 1737-1745.

21. P. Zhao, G. Liu, C. Zhao, A matrix-valued wavelet KL-like expansion for wide-sense stationary random processes, IEEE Trans. Signal Process. 52 (2004), no. 4, 914-920. 
Department of Mechanics and Mathematics, Oles Honchar Dnipro National University, Gagarin aV., 72, Dnipro, 49010, Ukraine

E-mail address: evgturchyn@gmail.com

Received ??.??.2019 